\title{
"Como sin querer la cosa". Insinuaciones e indeterminación en los encuentros entre esmeralderos y esmeraldas en Colombia
}

\author{
"As if without wanting to". Insinuations and indeterminacy in encounters \\ between esmeralderos and emeralds in Colombia
}

\author{
Vladimir Caraballo Acuña* \\ Instituto Colombiano de Antropología e Historia, Colombia
}

DOI: $10.22380 / 2539472 X .2046$

\begin{abstract}
RESUMEN
En el contexto de la formalización de la minería en Colombia, mineros artesanales, comerciantes y talladores de esmeraldas suelen enfatizar la indeterminación en sus encuentros con ellas y con sus cualidades. En lugar de acechar para extraer, de planear para tallar o de estandarizar para vender, en general privilegian la impredecibilidad de los encuentros y la espectralidad de las piedras. Por ello, lo que hacen es diseñar insinuaciones específicas de las cuales los encuentros interruptivos pueden surgir de manera indeterminada. En sus palabras, hay que buscar, tallar y vender esmeraldas "como sin querer la cosa". En este artículo propongo entender estas insinuaciones como prácticas lingüísticas y no lingüísticas que los esmeralderos usan para encontrarse con las esmeraldas y para crear la indeterminación como una teoría de su mundo que interrumpe el pensamiento económicopolítico de la formalización. Propondré que "como sin querer la cosa" nos da, como lo hace una guaca, la posibilidad de un pensamiento indeterminado erigido desde interrupciones y sorpresas. A este pensamiento, cuya naturaleza intento emular en la estructura misma del artículo, lo llamaré pensamiento esmeralda.
\end{abstract}

\begin{abstract}
$\overline{\text { ABSTRACT }}$
In the context of formalization of mining in Colombia, artisanal miners, traders, and emerald carvers often emphasize indeterminacy in their encounters with emeralds and their qualities. Instead of stalking to extract, planning to carve, or fixing to sell, they privilege the unpredictability of these encounters. What they do is to design specific insinuations from which interruptive encounters can indeterminately emerge. In their words, emeralds must be sought, carved, and sold "as if without wanting to". In this paper, I propose to understand these insinuations as linguistic and nonlinguistic practices that esmeralderos use to encounter with the emeralds and create indeterminacy as a theory of their world that besiege politic-economic thought of mining formalization. I propose that "as if without wanting to" gives us the possibility of an indeterminate thought constituted by interruptions and surprises. I call this thought, whose nature I try to emulate in the very structure of the article, pensamiento esmeralda.
\end{abstract}

Keywords: indeterminacy, insinuations, emeralds, linguistic anthropology.

Palabras clave: indeterminación, insinuaciones, esmeraldas, antropología lingüística.

1 vcaraballo@icanh.gov.co; vcaraballoa@gmail.com / https://orcid.org/0000-0003-2736-6531 
La indeterminación está [...] siempre abierta al horizonte y, en este sentido, es inherentemente creativa y liberadora. Comentario de Thomas Csordas a Abreu (2019)

Lejos de significar el fracaso del diálogo [la interrupción] podría llegar a ser la condición para la comprensión y el entendimiento [...] La interrupción de la relación, una cierta relación de interrupción, el suspenso de toda mediación. Derrida (2003, 19-20)

Hoy en día, nadie debe empecinarse en aquello que "sabe hacer". En la improvisación reside la fuerza. Todos los golpes decisivos habrán de asestarse como sin querer. Benjamin $(1987,21)^{1}$

\section{Introducción}

En Colombia, los encuentros entre esmeralderos (como llamaré de manera genérica a mineros y mineras artesanales, talladores y talladoras, y comerciantes) y esmeraldas están poblados de contradicciones, paradojas y ambigüedades. Más específicamente, es posible decir que los esmeralderos han diseñado prácticas concretas no solo para crear esas contradicciones, sino para mantenerlas irresueltas o resolverlas de manera provisional. Así, por ejemplo, los guaqueros (mineros artesanales) sostienen una delicada disposición potencial entre atención e indiferencia para que la esmeralda "se le aparezca" a uno entre el barro y las rocas; así, los talladores toman medidas precisas de los tamaños y formas de las piedras, no para domesticar su compleja materialidad, sino para que, por ejemplo, las tonalidades verdes surjan excediendo esas mismas medidas; y, de igual forma, cuando guaqueros, talladores y comerciantes fijan esos tonos en categorías cromáticas específicas, al mismo tiempo se encargan de decir que es imposible fijarlos porque siempre se están moviendo.

Los esmeralderos resumen estas ideas con un conjunto de frases diferentes, recurrentes y todas referidas a una misma forma de hacer las cosas: "como por no dejar”, “como sin querer” o "como sin querer la cosa”. En términos generales, para muchos de ellos, hay que buscar, tallar y comerciar esmeraldas "como sin querer la cosa”.

1 Le debo el hallazgo de esta frase de Benjamin a Andrea Murillo. 
Aunque en Colombia estas expresiones pueden referirse a un encubrimiento, a un simulacro diseñado para esconder una intención velada, en el mundo de las esmeraldas se relacionan con una forma de hacer que crea un espacio suspendido entre hacer y no hacer, entre decir y no decir: encontrar sin buscar, medir para exceder, fijar sin fijar. Hacer como sin querer la cosa es una forma de mantener irresueltas paradojas y contradicciones como estas; mejor aún, es una forma de resolverlas provisionalmente para no extinguirlas, de resolverlas y mantenerlas irresueltas al mismo tiempo. De la provisionalidad, de la irresolución, las esmeraldas y sus cualidades aparecen no como productos sino, en muchas ocasiones, como sorpresas.

Estas prácticas de talladores, mineros y comerciantes tienen un espesor político particular en la Colombia contemporánea: la formalización estatal de la minería. La formalización ha consistido en un conjunto de mecanismos orientados a garantizar la adhesión del país a la Organización para la Cooperación y el Desarrollo Económicos (OCDE), para su mejor posicionamiento en la inversión extranjera a través del diseño de economías transparentes y materias primas trazables. En este contexto, los funcionarios estatales han diseñado diferentes dispositivos de estandarización con el propósito de categorizar, contabilizar y organizar las prácticas de los esmeralderos: la difusión de la precisión como forma privilegiada de tallar, la certificación del origen de las piedras, la medición de la capacidad económica de los comerciantes, el control del número de piedras que cada guaquero ${ }^{2}$ puede comercializar, entre otros. Para muchos guaqueros, mineros y talladores tradicionales, la formalización ha implicado el aumento del monopolio imperante desde hace varios años (Caraballo Acuña 2019).

A pesar de lo que pudiera pensarse, la relación entre indeterminación y estandarización no ha sido binaria. Al contrario, los encuentros de los esmeralderos con los funcionarios y los dispositivos de estandarización suelen ser similares a los encuentros con las esmeraldas: en ellos, en lugar de la oposición directa, emerge una suerte de incomunicación hecha de interrupciones que nos permite imaginar, de manera más optimista, una economía política minera dispuesta a aprender de los encuentros esmeralderos, dispuesta a abrazar lo que la interrumpe y la puede sorprender creativamente.

2 En Colombia la palabra guaquero es usada con distintos significados. En el mundo esmeraldero, se refiere a mineros artesanales que buscan esmeraldas entre el barro de las laderas y las riberas de las quebradas usando palas y picos. En ocasiones, también se emplea para referirse a quienes ingresan subrepticiamente a túneles de empresas mineras abriendo túneles alternos que conectan con los principales. En cualquiera de los casos, los esmeralderos suelen aludir al origen chibcha de la palabra guaca para invocar con ella la ancestralidad de su actividad. 
En este artículo describo cómo, en el marco de la formalización minera, algunas prácticas de mineros artesanales, talladores y comerciantes pueden entenderse como insinuaciones, es decir, como prácticas que les permiten, al mismo tiempo, encontrarse con las esmeraldas y sus cualidades, declarar que esto solo es posible en medio de la indeterminación, y crear esa indeterminación como una teoría de su mundo. Sugeriré que las insinuaciones esmeralderas y el conocimiento presente en ellas pueden extenderse para entender casos similares en contextos muy distintos y, más aún, ayudarnos a fundar un pensamiento que abrace las interrupciones y las sorpresas. A este pensamiento lo llamaré pensamiento esmeralda.

Para desarrollar estos argumentos procederé de la siguiente manera: en la primera parte aprovecho la narración de José Emiro ${ }^{3}$, un amigo guaquero, sobre sus enguacadas, para mostrar la disposición ambigua, paradójica, entre la atención y la indiferencia, que los guaqueros deben asumir para insinuar la posibilidad de encontrarse con las esmeraldas. En la segunda parte sugiero que esta disposición puede ser entendida como un conjunto de insinuaciones, es decir, prácticas lingüísticas y no lingüísticas que crean la posibilidad de la aparición de esmeraldas valiosas y construyen las reglas mismas de sus encuentros con ellas; para lo anterior, analizo en detalle las formas poéticas de la narración de José Emiro y sugiero que, en sus historias, los guaqueros construyen a las esmeraldas como apariciones y a sus encuentros con ellas como eventos impredecibles. En la tercera parte muestro cómo estas insinuaciones guaqueras se relacionan con otro tipo de insinuaciones: las denominaciones ambiguas que utilizan los talladores y los comerciantes para categorizar los tonos verdes de las esmeraldas; a través de su estructura poética, sugiero que los esmeralderos declaran la fijación de las tonalidades y, al mismo tiempo, su imposibilidad. En la cuarta parte recojo estas insinuaciones para lanzar el centro de la propuesta analítica y para iniciar un cambio en el tono del documento: partiendo de las insinuaciones mencionadas sugeriré que, a través de ellas, los guaqueros, talladores y comerciantes crean una teoría de mundo basada en la indeterminación, que recientemente ha comenzado a asediar el pensamiento económico político de la formalización minera. Cierro el documento proponiendo la posibilidad de un pensamiento-esmeralda que busco emular en la escritura misma del texto: un pensamiento antropológico, económico y político basado en la potencialidad de estados intermedios, atenuados, entre la estandarización y el exceso. Así, si las primeras cuatro partes dan forma a una textura acotada, las últimas dos la 
desdoblan y extienden ensayísticamente con el objetivo de presentar la potencialidad como resultado estético y analítico de la operación.

\section{Encuentros entre el barro}

Permítanme ser convencional e iniciar con la manera como los guaqueros se encuentran con las esmeraldas. En Colombia, encontrarse con una esmeralda valiosa se llama “enguacarse”. El uso más frecuente de este término hace referencia a un guaquero que se enguaca mientras camina por las laderas de las montañas o las riberas de las quebradas del occidente de Boyacá. Pero el término también se utiliza para hablar de un tallador que durante el proceso de talla se encuentra con un efecto óptico no previsto, un brillo fulgurante, una tonalidad fascinante o una hermosa combinación de inclusiones (componentes gaseosos, sólidos y líquidos que constituyen el interior de las piedras). También se emplea para hablar de un comerciante que logra vender una esmeralda a un precio alto que jamás sospechó o de uno que logra comprar una a un módico precio que, igualmente, nunca imaginó. Guaqueros, comerciantes y talladores, algunas cuantas veces en sus vidas, en broma o en serio, han dicho de sí mismos "me enguaqué” o, de algún conocido, “se enguacó”.

Aunque sea posible sospechar la posibilidad de estos encuentros, enguacarse siempre es asombrarse, sorprenderse. Por eso, los esmeralderos suelen hablar de "encontrarse con una esmeralda" en lugar de "encontrar una esmeralda": enguacarse es siempre disponerse para el asombro y la sorpresa y, como veremos, dejarse tomar por ellos: dejarse tomar por sorpresa. Ni la esmeralda entre el barro ni el brillo en la talla son predecibles, esperables ni susceptibles de ser diseñados. Uno no encuentra una esmeralda o un color exuberante porque los esté buscando; se encuentra con ellos porque se le aparecen. Son, para decirlo en términos que irán tomando forma más adelante, parecidos a espectros: entidades suspendidas entre la presencia y la ausencia cuya forma de habitar es la interrupción (Barad 2010; Derrida 1995; Tsing et al. 2017).

Así, por ejemplo, para los guaqueros enguacarse es solo posible si se construye una suerte de estado suspendido en el que una esmeralda puede aparecer. Los guaqueros de Muzo, el municipio más representativo de las esmeraldas colombianas junto con Chivor y Coscuez (en la región occidental del departamento de Boyacá), suelen realizar caminatas en las laderas de las montañas y las riberas de las quebradas disponiéndose al encuentro con una “chispita”. El siguiente es el fragmento de una conversación que tuve con José Emiro sobre sus guacas: 
-José Emiro: Mis guacas casi la mayoría han sido de ojo, andando, andando, rompiendo piedras [...]

-Vladimir: ¿Y sí ha encontrado esmeraldas así, solo andando y mirando?

-J. E.: ¡Lógico! ¡Claro! Lo que pasa es que uno no debe ser negativo. Debe ser positivo, tener positivismo. Ir con esa ilusión, con ese ánimo, con ese deseo, con ese gusto: "hermano, yo me voy a enguacar. Hoy o mañana". $\mathrm{Y}$ con ese pensamiento me voy. O hay otra: tampoco puede uno ponerse a pensar tanto en eso. Mire, si yo me pongo a contarle lo de mis guacas... eso es para risas. Dios y suerte. Grábese eso: Dios y suerte. Un día le dije a un amigo que nos fuéramos por allá arriba para El Repollal. Yo llevaba un pantalón casi nuevo, azul, y una camisa. Yo nunca me ha faltado mi pico. Nos fuimos con el man para arriba. Y a lo que llegamos arriba el man se cansó y dijo que se iba para otro lado porque ya iba cansado por el sol. Le insistí: “marica, camine, hombre, vamos”. La esmeralda hay un momento como que lo lleva a uno, lo llama. Le dije: "camine, hombre”. Que no, dijo. Me dijo que más bien le prestara la pala. Y yo le dije que bueno, que si no iba pues bueno... que se llevara entonces la pala. Y arranqué yo para arriba con mi pico. Cuando llegué y vi una manguera ahí botada... una manguera de dos pulgadas, botando el chorro de agua. Y me puse con el pico a hacerle así [escarbando]... ¡como por no dejar! Y joda y joda con el pico. Y ipaannn, hijueputa! Ahí me la encontré. Dios bendito, alabado santo, Señor, Virgen santísima...

-V.: Muy de buenas usted.

-J. E.: Así me las encontraba yo siempre. Tin, tin, tin, y ahí me las encontraba. Echando pala no he sacado lo mismo que así andando [...] La esmeralda mía siempre ha sido de rapidez, no de tanto tiempo, ni de ponerse a dedicarse todo el día: dele pa'rriba, dele pa’bajo, dele pa'llá. ¡No! Si usted está para echar pala, para sacarla con la pala, mete la pala y sacó la esmeralda y listo, se acabó [...] En el momento menos pensado se le aparece. No hay que andar pensando en eso todo el tiempo: porque la esmeralda siente el estresamiento, la ansiedad, y se esconde. Mientras más demuestre, menos la encuentra. La esmeralda, pienso, es como una tranquilidad, una frescura. Y a veces lo llama a uno: "veeeenga”. La esmeralda no es para matarse trabajando. (Comunicación personal, 2018)

Sería imposible contar una enguacada sin las tonalidades y expresiones usadas por José Emiro: sin el suspenso, sin el “paaaannn, hijueputa”, sin la sorpresa. Un enguacado ve interrumpida su vida y las de los demás a través de un derroche que usualmente dura poco (Brazeal 2016; Páramo 2011; Uribe 1992). $\mathrm{Y}$ las historias sobre esas interrupciones son contadas también como sorpresas, como chispas llenas de un suspenso que, si se resuelve, lo hace de manera 
indeterminada, es decir, sin resolverlo en una cadena lineal de causas y efectos. ¿Por qué se enguaca José Emiro? ¿Cómo ocurre su encuentro?

Luego retornaré a su narración para mostrar cómo, a través de sus historias, los esmeralderos construyen una teoría de su mundo (ver Lepselter 2016). Por ahora me interesa llamar la atención sobre que, según nos dice José Emiro, enguacarse es solo posible cuando la persona sostiene un delicado equilibrio entre deseo e indiferencia. Para él, como para todos los guaqueros con quienes he hablado, si uno cede al deseo, "la esmeralda siente el estresamiento, la ansiedad, y se esconde” — para el caso del oro ver Jaramillo y Figueroa (2019)—. A mí, por ejemplo, me conminaban a esa relajación atenta cuando me veían caminando con la mirada clavada sobre el suelo y los ojos abiertos "de par en par", como si "mientras más los abriera más grande fuera la esmeralda que podía encontrar”, decían burlonamente. Si ocurre lo contrario, es decir, si la persona logra disponer su cuerpo a eso que ocurre cuando está atento e indiferente al mismo tiempo, "en el momento menos pensado se le aparece”. Disponerse a esta aparición consiste en una búsqueda que debe renunciar a buscar y una atención que debe ser distraída. Es una disposición ontológicamente contradictoria, paradójica (sobre un tipo de atención similar ver Abreu 2021, 80-105).

Lo que hacen los guaqueros cuando caminan en el barro y cuando rompen pequeñas rocas para dilucidar una chispa verde no es buscar algo que está oculto; no es acechar. Para encontrarse con la esmeralda, como dice José Emiro, hay que hacer "como por no dejar”; hay que hacer “como sin querer la cosa”. Más adelante volveré a los detalles de esta expresión.

Y lo que uno se encuentra "como por no dejar" es una chispa verde (ver Rose 2017). Una vez vista, el guaquero debe mantener la mirada fija en ella, no parpadear, doblar las rodillas, estirar un brazo y un par de dedos rápidamente, pero con cuidado para que, como dicen, la esmeralda no "se le desaparezca". El guaquero, en este sentido, debe disponer su cuerpo y su mente para la aparición; es así como se prepara a ser tomado por sorpresa. Igual que la disposición previa necesaria para el encuentro, mantenerlo vivo implica un delicado equilibrio entre movimiento y detención, una suerte de suspensión del cuerpo, de creación de un espacio intermedio, potencial.

Y es que, en efecto, las esmeraldas, como escuché muchas veces, se desaparecen fácilmente. Si usted no la agarra en el momento, solían advertirme, si usted parpadea, dela por perdida. Sea entre el barro y el agua, sea en su mano, sea cuando se cae y usted cree saber dónde se posó, sea en el bolsillo de su camisa, una esmeralda puede desaparecer de repente. Son muchas las historias: a muchos guaqueros, talladores y comerciantes una esmeralda se les ha desaparecido 
alguna vez en su vida. Las esmeraldas, como dijera Valero (2008), siempre "tienen un misterio" (222). Encontrarse con ellas tiene algo de espectral.

Pero, entonces, si encontrarse lo que esperan/no esperan no depende de ellos, ¿qué es lo que pueden hacer los guaqueros?, ¿en qué consiste esa delicada disposición?, ¿cómo puede uno disponerse a la sorpresa? (Messeri 2017). Lo que quiero sugerir es que los guaqueros y, como veremos, los talladores y los comerciantes, hacen algo muy específico: insinuar.

\section{Insinuaciones, impredecibilidad y apariciones}

Caminar, mover la pala de cierta manera, lavar la tierra, romper las rocas con un pico, inclinar el cuerpo, estirar los dedos son insinuaciones; también lo es el delicado ensamblaje entre los cuerpos de los talladores, las máquinas de talla y la materialidad de las piedras (Caraballo Acuña 2021); y también lo es la manera como declaran precios que, en lugar de fijar la relación entre una mercancía y una cantidad específica de dinero, crean la posibilidad de que un precio pueda surgir, aparecer (ver Guyer [2004] sobre un tratamiento similar de los precios en África atlántica). Son insinuaciones, es decir, sugiero, prácticas a través de las cuales las personas crean la posibilidad de que algo ocurra - en lugar de producirlo directamente-y, al mismo tiempo, reflexionan y dicen algo sobre lo que están haciendo.

Las insinuaciones suelen ser analizadas como delicadas estrategias comunicativas en las que el insinuador manipula la relación entre lo dicho y lo no dicho para cubrir su intención frente a un interlocutor (Bertuccelli Papi 2014; ver también Reyes 2004). Entendidas de esta manera, las insinuaciones parten de la distinción entre individuos discretos con intenciones claramente definidas que pueden ser mostradas u ocultadas.

Pero, aunque los esmeralderos suelen reconocer que las esmeraldas son voluntariosas, que deciden a quién aparecérsele, que eligen, que llaman, con sus insinuaciones no buscan manipularlas ni convencerlas subrepticiamente de algo ni, mucho menos, ocultar su responsabilidad (para una discusión sobre la distribución de la responsabilidad en este contexto ver Caraballo Acuña [2019]); los guaqueros no se relacionan con ellas como entidades cuyas intenciones puedan ser discretamente definidas y manipuladas. Enguacarse no depende solamente de la voluntad de la piedra, de Dios o de la suerte; depende de eso pero, 
sobre todo, de algo más; de algo más que no es posible determinar (ver A. A. Johnson [2020] sobre una relación similar entre pescadores del río Mekong y entidades espectrales) ${ }^{4}$.

Lo que quiero sugerir es que, con sus insinuaciones, los guaqueros construyen a las esmeraldas no solo como entidades voluntariosas, sino como apariciones y a sus encuentros, como eventos impredecibles. Y no lo dicen explícitamente, sino de manera implícita, en la forma como hablan de ello. Para demostrar esto quiero centrarme, ahora sí, en los detalles de la narración de José Emiro ${ }^{5}$.

Hay un conocimiento muy específico elaborado implícitamente en la historia que narra. En primer lugar, me interesa llamar la atención sobre los distintos niveles de narración con los que construye su historia -Bajtín ([1975] 1989) y Hill (1995)—. Específicamente, José Emiro alterna permanentemente tres niveles: los encuentros personales que ha tenido con las esmeraldas, a través del uso de unidades lingüísticas personales como "yo”, "mis”, "me”; los comentarios que hace acerca de las características de esos encuentros, con el uso de expresiones como "la esmeralda mía siempre ha sido..." o "eso es para risas"; y comentarios acerca de los encuentros que cualquier persona puede tener con las esmeraldas, con el uso de unidades más generales, como "usted" o "uno", en oraciones como “si usted está para...” o “como que lo llama a uno”. José Emiro cuenta su historia narrando sus encuentros y, en turnos, teorizando sobre ellos y sobre los de otros.

En segundo lugar, a partir de este dialogismo, el guaquero construye su conocimiento de dos formas: de manera explícita, narrando su encuentro José Emiro dice que enguacarse es, como ya advertí, una combinación de “ilusión, ánimo, deseo, gusto”, por un lado, y de “tranquilidad y frescura”, por otro; además, dice que los encuentros dependen de "Dios y suerte"; afirma que dependen también del "llamado" de las esmeraldas; y que los encuentros no están determinados por el trabajo, sino por todo lo anterior. Eso lo dice José Emiro explícitamente. Pero ¿qué dice la forma en la que habla? Lo que quiero mostrar es que, implícitamente, en la configuración poética de su práctica lingüística, dice algo mucho más

4 Agradezco a uno de los evaluadores por sugerirme la necesidad de hacer este énfasis.

$5 \quad$ Entiendo que "el lenguaje" no es distinto a "la práctica", como si se tratara de reinos separados; más bien, sigo a distintos antropólogos y antropólogas lingüistas al decir que el lenguaje es un tipo de práctica que debe ser analizada con la misma rigurosidad que cualquier otra. Como tal, está constituida y da forma a específicas materialidades (Harkness 2014), espaciotemporalidades (Dick 2010), texturas (Jakobson 1986) y relaciones ideológicas (Schieffelin, Woollard y Kroskrity [1998] 2012). "Recoger los conceptos en la vida" es posible también, contrario a lo que dijo el antropólogo colombiano Luis Guillermo Vasco, tomándonos absolutamente en serio las decisiones lingüísticas de las personas (Vasco 2002; ver también Anzola Rodríguez 2017). 
complejo que la simple articulación de voluntades. Específicamente, José Emiro usa tres oraciones que permiten entender lo que quiero decir:

Así me las encontraba yo.

En el momento menos pensado se le aparece.

Como por no dejar.

Aquí estoy siguiendo la importancia de prestar atención a los ritmos y texturas de las historias que escuchamos en campo (Tsing y Ebron 2015). Más específicamente, sigo la posibilidad de que las prácticas lingüísticas sean entendidas como prácticas rituales en las que los detalles mismos del aquí-y-ahora construyen las reglas que las hacen posibles (Silverstein y Urban 1996). En lugar del lenguaje como un conjunto de relaciones semióticas estandarizadas extraíbles de sus usos particulares (los sonidos, los acentos, las texturas), tal y como sugirió Saussure, se trata de un lenguaje comprensible solamente en-uso. En lugar de entender las formas de hablar como simples aplicaciones automáticas de estructuras lingüísticas previas, es necesario ver en ellas decisiones lingüísticas que tomamos en el curso mismo de nuestras interacciones.

En este sentido ritual, con el término metapragmática Michael Silverstein (2004) se ha referido a la manera como, a través de ciertas prácticas lingüísticas, las personas dicen algo y, simultáneamente, dicen algo acerca de lo que dicen, de cómo lo dicen y, por esa vía, del lenguaje mismo que les permite hacer todo lo anterior. Más específicamente, prestando atención a distintas "pistas" o formas poéticas de las prácticas lingüísticas (Jakobson 1986), se puede derivar el conocimiento que las personas construyen acerca de ellas mismas (Bauman y Briggs 1990). Es como observar un juego del cual no se conocen las reglas para, a partir de sus ritmos, sus repeticiones, sus interrupciones, derivar las reglas de funcionamiento que, sin duda, exceden siempre aquellas establecidas en un posible manual.

Lo que creo, entonces, es que las tres oraciones, que resuenan con el uso frecuente que hacen de ellas guaqueros, talladores y comerciantes, constituyen justamente pistas del conocimiento que José Emiro y los demás construyen sobre su relación con estos minerales. A la tercera oración volveré más adelante. Por ahora quiero fijarme en las dos primeras.

Si, siguiendo esta línea, pensamos esta forma de hablar como un conjunto de elecciones que José Emiro toma conscientemente, es posible ver dos cosas: primero, que en la primera oración evita decir algo como "así las encontraba yo”; hablando de esta forma, el guaquero usaría la forma con la que comúnmente nos referimos a algo que hemos estado buscando y que terminamos por encontrar, es decir, a la relación entre una búsqueda y un hallazgo. Pero, en su lugar, 
decide usar otra forma que, como dije antes, no es para nada casual: "así me las encontraba yo”. Hablando de esta manera, en una vía contraria a la anterior, José Emiro se decide por una fórmula a la que solemos acudir para hablar de encuentros casuales, no planeados, sorpresivos: como dije, encontrarse con algo es distinto a encontrar algo. Hablando así, sugiero, José Emiro está enfatizando que su encuentro, sus encuentros, son sorpresivos. Los guaqueros no encuentran esmeraldas, sino se encuentran, de manera impredecible, con ellas.

Y algo similar ocurre en la segunda oración, cuando lanza una teorización ya no sobre sus encuentros, sino sobre los encuentros de un "usted" que puede ser cualquier persona. Si en la primera oración habla del carácter impredecible, en la segunda evita decir algo como "en el momento menos pensado se la encuentra” (que sería concordante con la primera oración) y se decanta en su lugar por decir algo más radical: que uno ya ni siquiera se encuentra las esmeraldas, sino que las esmeraldas “se le aparecen”. La expresión, de nuevo, no es casual y, al contrario, se repite una y otra vez en las historias que los guaqueros cuentan sobre sus enguacadas.

Articulando la narración de su encuentro, los comentarios sobre sus encuentros en general, los comentarios sobre los encuentros que cualquier persona puede tener con las esmeraldas, y unas formas poéticas muy detalladas, José Emiro está construyendo el carácter impredecible de los encuentros y el carácter espectral de las esmeraldas.

Por supuesto, esto no solo ocurre en las prácticas lingüísticas. Tampoco ocurre solamente con los guaqueros. Caminando entre el barro, picando rocas, moviendo sus palas de cierta manera, mirando sin intensidad, pero atentos, los guaqueros disponen su cuerpo y su atención a ese limbo en el que, al mismo tiempo, esperan algo, no saben lo que esperan (más allá, por supuesto, de una esmeralda), no desean saberlo y se prestan a ser tomados por sorpresa. E, igual que los guaqueros solían llamarme a la distensión mientras caminaba en el barro, en muchas otras ocasiones lo hicieron también los talladores al verme tallar con prisa o, al contrario, con parsimonia, con aburrimiento. Y lo mismo hicieron los comerciantes cuando vieron que intentaba fijar un precio de manera apresurada, sin esperar a que el juego de declaraciones propio del regateo tuviera lugar. En la guaquería, la talla y el comercio, cada vez que veían que yo intentaba presionar un final, predecir lo que podía ocurrir, me llamaban a disponerme a la sorpresa, a la indeterminación, a la posibilidad de que algo relativamente inesperado ocurriera. En todos los casos, lingüísticos y no lingüísticos, guaqueros, talladores y comerciantes saben disponerse a través de las insinuaciones a esa suerte de estado intermedio y potencial del cual puede surgir una aparición. De nuevo: ¿por qué se enguacan José Emiro, un tallador o un comerciante? Por algo 
que no puede ser determinado y ante lo cual solo pueden disponerse haciendo insinuaciones.

Esta disposición, en mi opinión, es fundamental. Lo es porque nos ofrece la oportunidad de ver que la relación impredecible y espectral con las esmeraldas interrumpe, como veremos más adelante, un pensamiento propiamente económico hecho de entidades discretas que habitan el espacio de manera también discreta. En este sentido, mi propuesta es distinta al enfoque usual en los análisis provenientes de la sociología y la ciencia política que ven las esmeraldas (o cualquier otro "recurso") como objetos cuyo valor intrínseco define los conflictos por su apropiación (Gutiérrez Sanín y Barón 2008; Leiteritz y Riaño 2018; Parra 2021; cfr. Kneas 2018; Richardson y Weszkalnys 2014; Shever 2020).

Más específicamente, me interesa distanciarme de manera enfática de aquellas propuestas que ven la ambigüedad y la indeterminación esmeraldera como una simple "creencia ilusoria” de los guaqueros producida por relaciones económicas “estructurales” (Parra 2021, 15). Así, en lugar de explicarla como el producto de "una profunda ignorancia de los procesos efectivos de reconocimiento, comercialización de la cadena de producción [de parte de] la gran masa de guaqueros y de sus familias” (15), me propongo preguntarme qué pensamiento, por derecho propio, expresan en la manera como se encuentran con las esmeraldas; ese pensamiento, en lugar de un desfase ignorante del mundo económico, nos ayuda a comprender, de hecho, los límites de este (Johnson 2020, 12-13; Walsh 2004).

Para terminar de redondear este argumento volveré más adelante a la expresión "como por no dejar” o "como sin querer la cosa”. Pero, antes de ello, quiero centrarme en otro conjunto de insinuaciones también de tipo lingüístico: la declaración de las tonalidades de verde de las esmeraldas. Como veremos, también allí guaqueros, talladores y comerciantes crean contradicciones para fundar la provisionalidad en sus encuentros con, esta vez, el color de las piedras.

\section{Tonalidades verdes}

La indeterminación potencial de la que hablo es creada y sostenida por talladores y comerciantes de esmeraldas que también deben disponerse al asombro. Entre otros momentos, esto ocurre cuando unos y otros deben decirse de qué tono de verde es una esmeralda. Aunque no hay disputas acerca de que estas piedras son de color verde, el tono específico es un asunto delicado porque los tonos más 
saturados suelen ser altamente valorados en el mercado. Pero la delicadeza de las formas en que los esmeralderos declaran estos tonos no se explica solamente, de nuevo, por los intereses comerciales. Al igual que en la guaquería, los talladores, los comerciantes y los guaqueros han decidido que los tonos verdes solo pueden ser declarados de esa misma manera: como sin querer. Para ellos, hablar de los tonos de las piedras es solo posible contradiciéndose: fijar el tono de una esmeralda y, al mismo tiempo, decir que los tonos de las esmeraldas no se pueden fijar. Lo hacen todo el tiempo. Explícitamente dicen que, a diferencia de los diamantes o del oro, el color de las esmeraldas es tan complejo que no es posible estandarizar su denominación; pero, nuevamente, lo hacen también de manera implícita a través de la estructura poética de sus declaraciones. Veamos.

Los comerciantes establecen tres tonalidades en relación con el origen de las piedras: el color de las provenientes de Muzo ha comenzado a definirse como "verde Muzo" o "verde yerba”; las de Chivor, dicen, tienen un verde "más azuloso" o "más hacia el azul”; las de Coscuez, un verde "más amarilloso" o "más hacia el amarillo”. Por supuesto no me interesa sentar una posición acerca de si existen o no ciertas categorías cromáticas ni de su relación con determinadas unidades culturales (Young 2018); más bien, quiero mostrar, en una orientación más pragmática, aquello que los esmeralderos hacen con estas formas de denominación y, específicamente, lo que dicen con ellas acerca de la naturaleza de sus encuentros con los tonos de las esmeraldas. Fújense entonces en la semejanza poética que comparten estas categorizaciones: el uso de preposiciones de espacio (hacia), de adverbios de cantidad (más, menos) y de los sufijos oso u osa. ¿En qué se parecen estas unidades lingüísticas? Quiero argumentar lo siguiente: a través de ellas los esmeralderos fijan los tonos y, a la vez, dicen que esa fijación es posible solamente siendo provisional, es decir, que sostienen al mismo tiempo la fijación y el movimiento de los tonos. Veamos, solo como un ejemplo, la siguiente frase:

Esta [esmeralda] es más hacia el amarillo.

En otras ocasiones esta frase, típica en el mercado esmeraldero, es enfatizada con la siguiente forma:

Esta [esmeralda] es más como hacia el amarillo.

En el primer caso, los esmeralderos hacen dos cosas: comparar esmeraldas a partir del amarillo como cualidad compartida y graduar esa comparación a través del adverbio de cantidad “más”. En el segundo caso ocurre lo mismo, pero se agrega una atenuación operada por el “como” (y que, de hecho, veremos, cumple el mismo papel en "como sin querer la cosa”). Parece un caso típico de graduación de cualidades, pero quiero llamar la atención sobre algo que me parece 
más interesante: la inclusión de la preposición "hacia”. Pensemos, nuevamente, estas formas de hablar como elecciones lingüísticas que toman las personas para hacer algo; por ejemplo, veamos la diferencia implicada si los esmeralderos dijeran simplemente:

Esta es más amarilla.

¿Por qué no lo hacen? Es claro que, frente a esta oración, el uso del "hacia” les permite a los esmeralderos denotar el movimiento hacia un destino (en este caso el amarillo). Lo que quiero sugerir es que, a través de su inclusión, los esmeralderos declaran el tono $y$, a la vez, declaran que los tonos de los que hablan se están moviendo. Fijan un tono y al mismo tiempo, implícitamente, en la configuración misma de la oración, dicen que esa fijación no es más que una resolución provisional, posible e imposible, que mantiene viva la tensión entre movimiento y detención. Así, sostienen una tensión y la resuelven solo de manera provisional, sin extinguirla.

Esta convivencia entre la fijación y el movimiento en las formas de graduar cualidades no es nueva. El antropólogo lingüista Edward Sapir (1944) realizó un elegante estudio acerca de la fijación y el movimiento en las prácticas lingüísticas de graduación. Según él, por ejemplo, las operaciones lingüísticas en las que se establecen equivalencias implican siempre, implícitamente, un movimiento. Decir que algo es "igual a" algo consiste en establecer "un punto más o menos temporal de un pasaje”, dice, o un equilibrio entre el "más que” y el "menos que”; se trata, dice el autor, de "un punto de llegada” en una escala en la cual el término que debe ser graduado está “en permanente crecimiento o disminución” (Sapir 1944, 105; ver también Kockelman 2016; Nakassis 2016). En la frase "esta es más hacia el amarillo” ocurre algo similar, pero enfatizado con la inclusión de la proposición "hacia”. Lo que hacen con ello los esmeralderos es declarar que fijan, sí, pero que su fijación no es más que el momento provisional de un movimiento perpetuo hacia otro lugar. En este sentido, la frase es un gesto metapragmático que fija un tono y, a la vez, declara algo sobre la naturaleza de esa fijación y, más aún, sobre la naturaleza ambigua, entre fijación y movimiento, estándar y exceso, de los colores de las esmeraldas (cfr. Benjamin 2011; Taussig 2008).

Algo similar ocurre con otra de las fórmulas usadas pero esta vez a través de los sufijos oso y osa.

“Esta es amarillosa” o “esta es azulosa”.

Dos formulaciones claramente diferentes a:

“Esta es amarilla” o "esta es azul”. 
A pesar de que en términos gramaticales estos sufijos suelen usarse para aumentar la pertenencia a una cualidad (arcilloso/a, acuoso/a, caluroso/a), no es este el caso. “Amarillosa” y “azulosa” no se refieren simplemente a que las esmeraldas sean muy amarillas o muy azules; más bien, es posible decir que denotan el mismo movimiento de la preposición “hacia”. Siguiendo esta línea, nótese que no es casual que en ninguna declaración aparezcan el sufijo y la proposición al mismo tiempo; algo como “más hacia el azuloso” o “más hacia el amarilloso” generaría una sensación de redundancia. De cierta manera, el sufijo parece reemplazar a la preposición por hacer también referencia al movimiento perpetuo y a la provisionalidad de la fijación.

El caso es que, lo que quiero demostrar con este análisis un poco denso es que, a través del sostenimiento implícito de la contradicción entre movimiento y detención, los esmeralderos dicen algo acerca de su encuentro con los tonos de las piedras como una de las cualidades más valoradas en el mercado. Dicho en otras palabras, lo que en la guaquería es el sostenimiento suspendido de atención y deseo, de aparición y desaparición, de posibilidad e imposibilidad, es aquí el sostenimiento ambiguo de fijación y movimiento. Nuevamente, los esmeralderos no solo clasifican para intercambiar, sino, al mismo tiempo, dicen algo acerca de la naturaleza contradictoria del ejercicio mismo de clasificación (ver Tsing 2013).

\section{"Como sin querer la cosa": la indeterminación como teoría de mundo}

Permítanme ahora retornar a la relación entre insinuaciones y conocimiento esmeraldero. Cuando los guaqueros revuelven la tierra y rompen piedras con sus picos, y cuando cuentan historias sobre ello, no solo quieren insinuar la posibilidad de un encuentro, sino hablar de la espectralidad y la imprevisibilidad de estos, y de la disposición ambigua de su atención. Cuando los talladores, comerciantes y guaqueros definen la tonalidad verde de una esmeralda, no lo hacen solo para categorizarla y compararla, sino para decir, explícita e implícitamente, que sus colores son detención y movimiento a la vez y que su encuentro con ellos siempre es, al igual que su clasificación, provisional. Insinuando, los guaqueros, los talladores y los comerciantes se encuentran con las esmeraldas, reflexionan sobre los encuentros y nos dicen que todo ello puede ocurrir solamente en el limbo potencial y provisional creado por las insinuaciones que mantienen irre- 
sueltas distintas tensiones. Las insinuaciones son formas reflexivas de hacer y, al mismo tiempo, de contar algo sobre el hacer.

Justo antes de narrar el momento en que la esmeralda se le aparece, José Emiro dice que usaba su pico de una manera muy particular: "como por no dejar”. Como anuncié, los esmeralderos suelen resumir todas las insinuaciones de las que he hablado con fórmulas similares a esta y, de manera particularmente recurrente, con una específica: “como sin querer la cosa”. Encontrarse con las esmeraldas (en la minería, en la talla, en el comercio) es solo posible "como sin querer la cosa”. Creo que usando esta oración y otras similares los esmeralderos hacen algo distinto a lo que hacen con comentarios como "así me las encontraba yo", "en el momento menos pensado se le aparece” o "más hacia...”. Usadas una $\mathrm{y}$ otra vez por guaqueros, comerciantes y talladores, oraciones como esta son “pistas”, ya no solo de la imprevisibilidad y la espectralidad, sino de un nivel más grueso de teorización: de la indeterminación como una teoría sobre esos $\operatorname{contextos}^{6}$. En otras palabras, “como sin querer la cosa” resume un conocimiento muy complejo acerca de los encuentros que los esmeralderos tienen con las esmeraldas.

A propósito también de las guacas en Colombia (no solo esmeralderas), Suárez Guava escribió:

el carácter sistemático de los elementos relacionados en las narrativas sobre la riqueza enterrada en Aldana y en Murillo me obliga a considerar la posibilidad de que allí exista un tipo de conocimiento acerca del mundo. Otra forma de decirlo es que allí existe un mundo que emerge gracias a él. Ese conocimiento será, por ende, la teoría de ese mundo. $(2013,14)$

6 Hay fórmulas similares a "como sin querer la cosa" en otros lugares del mundo. Por ejemplo, la famosa fórmula lingüística de Bartleby el escribiente en la novela de Herman Melville, "I would prefer not to", es un claro ejemplo de la indeterminación. Como lo mostraron Agamben, Deleuze y Pardo (2000), la fuerza provocada por la famosa fórmula lingüística de Bartleby no radica en la resistencia ni en la obediencia del escribiente, sino en la potencialidad que funda. A través de su iterativa formulación, Bartleby renuncia al acto - en cuanto elección que elimina otras elecciones-y se sitúa en la indeterminación de lo que es y no es al mismo tiempo. María José de Abreu, de otro lado, se ha acercado a las interrupciones, las tautologías y las relaciones tecnológicas como prácticas usualmente autorreferenciales con ritmos propios que fundan estados intermedios, abiertos, cargados de potencialidad y no pocas veces articulados con lógicas neoliberales o autoritarias (Abreu 2021). En un contexto completamente distinto, Andrew Johnson (2020) ha mostrado el papel de lo ambiguo en la relación entre campesinos de Indonesia y el río Mekong a través del uso recurrente del "quizás" (maybe). Frente a un escenario extractivo que irrumpe en las relaciones locales, los pobladores del río suelen referirse a sus vínculos con él, con las empresas extractivas y con ellos mismos, usando permanentemente esta palabra. Johnson argumenta que de esta forma los habitantes evitan comprometerse con la firmeza de las afirmaciones y las negaciones para así mantener siempre abierta la posibilidad de que algo distinto ocurra, algo que no puede conocerse, pero que es posible que venga. 
Mi argumento es que, por medio del ritmo creado por las resonancias entre insinuaciones, encuentros y comentarios reflexivos, los esmeralderos han creado una teoría acerca de su propio mundo, una teoría de mundo basada en la indeterminación.

Con indeterminación simplemente quiero recoger lo dicho hasta aquí. Aunque los guaqueros saben que con sus movimientos buscan provocar la aparición de algo concreto, es decir, de una esmeralda valiosa, al mismo tiempo enfatizan que el origen de esa ocurrencia no puede ser determinado. A pesar de que talladores y comerciantes saben que necesitan declarar un tono específico para poder comparar, dicen también que, en últimas, ese tono es imposible de determinar. Por ello, esmeraldas y tonos suelen aparecer como interrupciones que, como nos enseña su etimología, rompen en pedazos la continuidad para poner un espacio entre ellos (Diccionario etimológico español 2020). Los encuentros con las esmeraldas y sus cualidades tienen lugar siempre en ese espacio creado por la provisionalidad. El limbo generado por estas interrupciones es un espacio indeterminado caracterizado por la recreación permanente de la posibilidad, tanto por lo que ocurre como por, de manera fundamental, la creación de la posibilidad misma de que algo ocurra (Abreu 2019) ${ }^{7}$.

El término indeterminación está relacionado con otros similares, como incertidumbre, ambigüedad e ilegibilidad. Son expresiones que suelen usarse para describir escenarios precarios definidos por la dificultad de construir coordenadas cognitivas, económicas y políticas estables; allí, la creatividad de las poblaciones se refiere a las formas para resolver la incertidumbre, para determinar las coordenadas (Calkins 2016; Cross 2014; Dolan, Huang y Gordon 2019; Tsing 2015; entre otros). En un volumen reciente acerca de la indeterminación como un tercer término entre el valor y el desecho, Alexander y Sanchez enumeran tres formas que puede tomar lo indeterminado: a) la falta de reconocimiento o de incorporación en un sistema de clasificación; b) la imposibilidad de definir futuros y direcciones; y c) la resistencia a sistemas totalizadores $(2019,3)$. El mundo esmeraldero nos enseña que la indeterminación puede ser deliberadamente recreada para garantizar los encuentros entre personas y otras entidades

7 En su libro Anatomización. Una disección etnográfica de los cuerpos, Santiago Martínez Medina (2021) se acerca al papel de las interrupciones en las relaciones entre estudiantes de medicina y cadáveres: "La impresión, si se relaciona bien, excede el aprendizaje anatómico. A los estudiantes no se los enseña a impresionarse y, por ende, los momentos que impresionan son individuales, eventuales y ocasionales" (Martínez Medina 2021, 223, énfasis en el original). Si algo nos enseñan los esmeralderos es, justamente, cómo las irrupciones, las impresiones, trascienden - como lo muestra la narración de José Emiro- la experiencia personal para erigirse en una teoría de sus encuentros con las esmeraldas; más aún, en una teoría de su mundo basada, en los términos de Martínez, en la capacidad de impresionarse. 
-minerales, en este caso-; los encuentros (con las esmeraldas, con sus tonos, con el dinero) ocurren, se determinan, pero siempre como paradojas, como eventos que no agotan la indeterminación ${ }^{8}$.

Con indeterminación me refiero también, entonces, a un fenómeno político. Y es en este sentido que la relación entre indeterminación y formalización ha excedido la simple oposición binaria. De cómo los funcionarios buscan extinguir la indeterminación a través de dispositivos de estandarización he comenzado a hablar en otro lugar (Caraballo Acuña 2021). Sin embargo, aunque sea material para otro momento, es necesario decir aquí que los encuentros entre los esmeralderos, los funcionarios y los dispositivos de formalización suelen estar marcados no por la resistencia a la que se refieren Alexander y Sanchez (2019), sino por el desespero de una comunicación imposible. En esos encuentros, los esmeralderos, si bien se muestran dispuestos a oponerse a los mecanismos de categorización, medición y comparación, la mayor parte del tiempo buscan interrumpir (en el sentido que aquí le he dado) el pensamiento económico político de la formalización. Los encuentros con los dispositivos de formalización y los funcionarios no agotan, como ningún otro de los encuentros que hemos visto, la indeterminación; al contrario, en muchos momentos suelen constituirse justamente como encuentros que, a pesar de los funcionarios mismos y de la sofisticación de los dispositivos, terminan inevitablemente por tomar la forma de la indeterminación.

Es esta presencia interruptiva la que ha asediado, para seguir el término con el que Derrida (1995) describió la forma de habitar de lo espectral, el pensamiento económico-político de la formalización minera. La interrupción que, como él mismo dice en uno de los epígrafes de este artículo, puede ser la condición misma para la comprensión y el entendimiento. Es el asedio de una presencia que, como ha ocurrido durante décadas (Páramo 2011; Uribe 1992), es imposible de ubicar porque, justamente, es la presencia propia de la paradoja, del sin querer.

Y esta relación entre la indeterminación y el pensamiento económico-político de la formalización me lleva a la parte final en la que sugiero extender lo que, entonces, pueden enseñarnos los esmeralderos, sus insinuaciones y su conocimiento, ya no solo a los funcionarios estatales, sino también a los antropólogos. Lo que pueden enseñarnos, sugiero, es la posibilidad de un nuevo pensamiento: un pensamiento esmeralda.

8 En este sentido, "como sin querer la cosa" incluye la intención expresada en la fórmula que en ocasiones le da continuación: "pero la cosa queriendo". "Como sin querer la cosa" no implica una renuncia al hacer, al querer algo, pero sí enfatiza una forma particular de hacerlo (agradezco a Juana Camacho este apunte). 


\section{Un pensamiento esmeralda}

Mi interés en este artículo no ha sido negar que las esmeraldas, efectivamente, de manera determinada, se encuentran, se tallan y se intercambian por dinero; sin encontrarlas, procesarlas y comercializarlas el mundo esmeraldero quizá no existiría. Esa historia, como he dicho, es contada de manera recurrente. Lo que he mostrado es que reducir la relación entre esmeraldas y personas a esto ocultaría todo lo que complica una relación meramente económica en el sentido descrito por Timothy Mitchell (2002): un conjunto de prácticas basadas en el cálculo a partir de entidades discretas, diferenciables y comparables las unas de las otras, y orientadas a definir relaciones de control — ver también Callon, Méadel y Rabeharisoa (2002)—. Mi propuesta ha sido que los detalles de lo que hacen los esmeralderos son pistas de una complicación que consiste en mantenerse en medio: conmensurables e inconmensurables al mismo tiempo, espectros y mercancías, economía y exceso, paradojas en lugar de resistencia, interrupción en lugar de relación binaria, indeterminación.

En este sentido, lo que hacen los esmeralderos puede ayudarnos a entender prácticas muy distintas a las suyas (ver nota al pie número 6), como la ambigüedad estética de los bailes zulú en medio de escenarios colonialistas (Meintjes 2017), la comprensión de los vínculos entre personas diagnosticadas de demencia y las “entidades ambiguas” que habitan sus mundos (Dyring y Grøn 2021), la indeterminación como característica de las "relaciones afectivas” (Stewart 2007), las relaciones con entidades no humanas que retan la continuidad espaciotemporal (Bubandt 2018) o la escritura etnográfica misma (Paper Boat Collective 2017; Tsing y Ebron 2015). En todos estos casos, las insinuaciones y la indeterminación, como aquí las entiendo, desempeñan papeles fundamentales.

Más que estos asuntos específicos, lo que podemos recibir de parte de la indeterminación esmeraldera es similar a lo que reciben los enguacados: la indeterminación como la posibilidad de un nuevo pensamiento. Lo que el mundo esmeraldero puede darnos no es la indeterminación como un objeto de estudio, como un problema etnográfico, como una presencia. Lo que nos da es la posibilidad de la indeterminación como pensamiento. Lo que podrían darnos las insinuaciones esmeralderas es la posibilidad de un pensamiento indeterminado, uno distinto al que piensa exclusivamente desde la definición (Simondon 2009).

Quiero terminar llamando a esta posibilidad pensamiento esmeralda: un pensamiento (antropológico, político, económico) inclinado irremediablemente a insinuar abrazando las interrupciones para habilitar siempre la aparición de la sorpresa, de lo asombroso. El pensamiento esmeralda se extiende, o debería 
extenderse, para fundar un pensamiento-chispa, un pensamiento-sorpresa que pueda cuestionar los límites de un pensamiento económico y de un pensamiento político basados en la estandarización escalable que eliminan como riesgoso lo que insiste en mantenerse ya ni siquiera particular, sino indeterminado.

En este sentido, volviendo a la relación con la formalización, vale la pena preguntar: ¿y si los funcionarios estatales aprendieran a encontrarse con las personas como los guaqueros se encuentran con las esmeraldas? ¿Y si esos funcionarios aprendieran a diseñar las políticas mineras como los talladores tallan sus piedras? Una política minera hecha de gestos, de dispositivos político-poéticos, de insinuaciones siempre abiertas a lo impredecible; capaz de dar forma, de diseñar, pero, al menos como principio, al menos como un faro ético, siempre de manera provisional (Caraballo Acuña 2021). Siguiendo a Isabelle Stengers, Kristina Lyons (2021) dice que la esperanza es un intento de sentir lo que se oculta en los intersticios o aquello que normalmente elude la descripción, puesto que las palabras más fácilmente disponibles suelen referirse a entidades estabilizadas: "la esperanza no significa esperanza respecto de una u otra cosa ni una actitud calculada, sino un intento de sentir y expresar con palabras una posibilidad de devenir” (Stengers citada en Lyons 2021, 169).

El pensamiento esmeralda puede también extenderse a la forma misma como hacemos antropología. Preguntémonos entonces: ¿y si, por ejemplo, siempre que hagamos etnografía la hacemos así: como sin querer la cosa? ¿Será posible, incluso, escribir etnográficamente como sin querer, como por no dejar? Hacer etnografía, por ejemplo, esperando y sin esperar a la vez, indiferentes y atentos; sin forzar finales y sabiendo que lo mejor vendrá siempre como una sorpresa a la que debemos disponernos sin afanes, pero sin parsimonia (Howe y Takaragawa 2017). Hacer trabajo de campo y escribir etnografía como sin querer la cosa implicarán siempre el interminable pulimiento del arte esmeraldero, que es el arte de saber insinuar; un arte que tiene la capacidad de determinar, de decidir, de comprometerse, pero siempre con la humildad de la provisionalidad, siempre con el compromiso de la potencialidad, de la posibilidad de que siempre algo nuevo ocurra ${ }^{9}$.

Este artículo, en este sentido, no es más que un gesto con el que he querido hacer declaraciones acotadas, pero solo con el objetivo de excederlas; en el que espero cerrar, pero con el único propósito de abrir. Lo que he querido no es otra cosa más que hacer una insinuación, así... como sin querer la cosa.

9 Para ampliar discusiones sobre la escritura etnográfica en un sentido similar a este, recomiendo las conversaciones del podcast Escritura etnográfica, de la Revista Colombiana de Antropología, disponible en el canal de YouTube del Instituto Colombiano de Antropología e Historia. 


\section{Agradecimientos}

Este artículo es producto de mi investigación doctoral en antropología y del trabajo de campo realizado entre Bogotá y el occidente de Boyacá entre el 2015 y 2019. Agradezco a los guaqueros (especialmente a José Emiro), talladores, comerciantes, gemólogos y funcionarios del Ministerio de Minas y de la Agencia Nacional de Minería que compartieron su trabajo y sus días conmigo. Agradezco las lecturas previas de María Isabel Galindo, Anne Johnson y Luis Alberto Suárez Guava. La investigación doctoral contó con los apoyos financieros del Consejo Nacional de Ciencia y Tecnología en México, del Consejo Latinoamericano de Ciencias Sociales, del Instituto Colombiano de Antropología e Historia en Colombia y de Latin American Studies Association en Estados Unidos.

\section{Referencias}

Abreu, Maria José de. 2019. "Medium theory; or, 'The war of the worlds' at regular intervals”. Current Anthropology 60 (5): 656-673. https://doi.org/10.1086/705345

-. 2021. The charismatic gymnasium. Breath, media, and religious revivalism in contemporary Brazil. Durham: Duke University Press.

Agamben, Giorgio, Gilles Deleuze y José Luis Pardo. 2000. Preferiría no hacerlo. "Bartleby el escribiente" de Herman Melville seguido de tres ensayos sobre Bartleby de Gilles Deleuze, Giorgio Agamben y José Luis Pardo. Madrid: Pretextos.

Alexander, Catherine y Andrew Sanchez. 2019. "Introduction: The values of indeterminacy”. En Indeterminacy: waste, value, and the imagination, editado por Catherine Alexander y Andrew Sanchez, 1-30. Nueva York: Berghahn Books.

Anzola Rodríguez, Juan Sebastián. 2017. Uno hace la finca y la finca lo hace a uno. Trabajo, conocimiento y organización campesina en una finca en Sucre, Cauca. Santander de Quilichao; Bogotá: Gente Nueva Editores.

Bajtín, Mijaíl. (1975) 1989. Teoría y estética de la novela. Madrid: Taurus.

Barad, Karen. 2010. "Quantum entanglements and hauntological relations of inheritance: dis/continuities, SpaceTime enfoldings, and justice-to-come”. Derrida Today 3 (2): 240-268. https://doi.org/10.3366/drt.2010.0206

Bauman, Richard y Charles Briggs. 1990. "Poetics and performance as critical perspectives on language and social life”. Annual Review of Anthropology 19: 59-88. http://www.jstor. org/stable/2155959

Benjamin, Walter. 1987. Dirección única. Madrid: Alfaguara. 
—. 2011. "A child's view of color”. En Early writings 1910-1917. Massachusetts: Harvard University Press.

Bertuccelli Papi, Marcella. 2014. “The pragmatics of insinuation”. Intercultural Pragmatics 11 (1): 1-29. https://doi.org/10.1515/ip-2014-0001

Brazeal, Brian. 2016. "Nostalgia for war and the paradox of peace in the Colombian emerald trade”. Extractive Industries and Society 3 (2): 340-349. https://doi.org/10.1016/j. exis.2015.04.006

Bubandt, Nils, ed. 2018. A non-secular Anthropocene: spirits, specters and other nonhumans in a time of environmental change. More-than-Human. AURA Working Papers. Vol. 3. Moesgård: Aarhus University. https://anthropocene.au.dk/fileadmin/Anthropocene/MORE_TH AN_HUMAN_vol.3.pdf

Calkins, Sandra. 2016. Who knows tomorrow? Uncertainty in Nort-Eastern Sudan. Nueva York; Oxford: Berghahn Books.

Callon, Michel, Cécile Méadel y Vololona Rabeharisoa. 2002. “The economy of qualities”. Economy and Society 31 (2): 194-217. https://doi.org/10.1080/03085140220123126

Caraballo Acuña, Vladimir. 2019. "Semiotic distribution of responsibility: an ethnography of overburden in Colombia's emerald economy”. Extractive Industries and Society 6 (4): 1040-1046. https://doi.org/10.1016/j.exis.2019.03.018

-. 2021. "Carving mining policies. Hints and interruptions in infrastructural design of emeralds and the mining formalization in Colombia”. Inédito.

Cross, Jamie. 2014. Dream zones. Anticipating capitalism and development in India. Londres: Pluto Press.

Derrida, Jacques. 1995. Espectros de Marx. El estado de la deuda, el trabajo del duelo y la nueva internacional. Madrid: Trotta.

-. 2003. Carneros. Buenos Aires: Amorrortu.

Diccionario etimológico español. 2020. http://etimologias.dechile.net/

Dick, Hilary Parsons. 2010. "Imagined lives and modernist chronotopes in Mexican nonmigrant discourse”. American Ethnologist 37 (2): 275-290. https://doi.org/10.1111/j.15481425.2010.01255.x

Dolan, Catherine, Juli Huang y Claire Gordon. 2019. "The ambiguity of mutuality. Discourse and power in corporate value regimes”. Dialectical Anthropology 45: 9-27. https://doi. org/10.1007/s10624-019-09569-y

Dyring, Rasmus y Lone Grøn. 2021. "Ellen and the little one: a critical phenomenology of potentiality in life with dementia”. Anthropological Theory (junio). https://doi. org/10.1177/14634996211010512

Gutiérrez Sanín, Francisco y Mauricio Barón. 2008. “Órdenes subsidiarios. Coca, esmeraldas: la guerra y la paz”. Colombia Internacional 67: 102-129. http://www.scielo.org.co/ scielo.php?script=sci_arttext\&pid=S0121-56122008000100006

Guyer, Jane I. 2004. Marginal gains. Monetary transaction in Atlantic Africa. Chicago: The University of Chicago Press. 
Harkness, Nicholas. 2014. Songs of Seoul. An ethnography of voicing in Christian South Korea. Berkeley: University of California Press.

Hill, Jane. 1995. "The voices of don Gabriel. Responsibility and self in a modern Mexicano narrative”. En The dialogic emergence of culture, editado por Dennis Tedlock y Bruce Mannheim, 97-147. Urbana; Chicago: University of Illinois Press.

Howe, Cymene y Stephanie Takaragawa. 2017. “Surprise”. Theorizing the Contemporary, Cultural Anthropology. https://culanth.org/fieldsights/surprise

Jakobson, Roman. 1986. Ensayos de lingüística general. Ciudad de México: Artemisa.

Jaramillo, Pablo y Giselle Figueroa. 2019. "La atracción de los recursos: los afectos materiales y financieros del oro entre Marmato y Londres”. Ponencia presentada en el Congreso Colombiano de Antropología, Universidad Icesi, Cali, 11-14 de junio.

Johnson, Andrew Alan. 2020. Mekong dreaming: life and death along a changing river. Durham: Duke University Press.

Kneas, David. 2018. "Emergence and aftermath: the (un)becoming of resources and identities in Northwestern Ecuador”. American Anthropologist 120 (4): 752-764. https://doi. org/10.1111/aman.13150

Kockelman, Paul. 2016. The chicken and the quetzal. Inconmensurate ontologies and portable values in Guatemala's cloud forest. Durham: Duke University Press.

Leiteritz, Ralf J. y Manuel Riaño. 2018. “Tras el corazón verde: los vaivenes del conflicto en la región esmeraldera de Colombia”. En Diferentes recursos, conflictos diferentes: la economía política del conflicto armado y la criminalidad en las regiones de Colombia, editado por Angelika Rettberg, Ralf Leiteritz, Carlo Nasi y Juan Diego Prieto, 293-340. Bogotá: Universidad de los Andes.

Lepselter, Susan. 2016. The resonance of unseen things. Poetics, power, captivity, and UFOs in the American Uncanny. Ann Arbor: University of Michigan Press.

Lyons, Kristina M. 2021. Descomposición vital. Suelos, selva y propuestas de vida. Bogotá: Editorial Universidad del Rosario.

Martínez Medina, Santiago. 2021. Anatomización. Una disección etnográfica de los cuerpos. Bogotá: Universidad de los Andes.

Meintjes, Louise. 2017. Dust of the Zulu. Gnoma aesthetic after Apartheid. Durham; Londres: Duke University Press.

Messeri, Lisa. 2017. "Resonant worlds: cultivating proximal encounters in planetary science”. American Ethnologist 44 (1): 131-142. https://doi.org/10.1111/amet.12431

Mitchell, Timothy. 2002. Rule of experts. Egypt, techno-politics, modernity. Berkeley: University of California Press.

Nakassis, Constantine. 2016. "Scaling red and the horror of trademark". En Scale: discourse and dimensions of social life, editado por Summerson Carr y Michael Lempert, 159-184. Berkeley: Unversity of California Press.

Paper Boat Collective. 2017. "Introduction: Archipelagos, a voyage in writing”. En Crumpled paper boat: experiments in ethnographic writing, editado por Anand Pandian y Stuart McLean, 11-28. Durham: Duke University Press.

Páramo, Carlos Guillermo. 2011. "El corrido del minero: hombres y guacas en el occidente de Boyacá”. Maguaré 25 (1): 25-109. https://revistas.unal.edu.co/index.php/maguare/article/view/26763 
Parra, Johanna. 2021. "El laberinto de las esmeraldas. Incertidumbres y dificultades de la minería en el Occidente de Boyacá, Colombia”. Inédito.

Reyes, Graciela. 2004. "Pragmática y metapragmática: la ironía lingüística”. En Actas del XIV Congreso de la Asociación Internacional de Hispanistas, coordinado por Isaías Lerner, Roberto Nival y Alejandro Alonso, 147-158. https://cvc.cervantes.es/literatura/aih/pdf/14/ aih_14_1_018.pdf

Richardson, Tanya y Gisa Weszkalnys. 2014. "Introduction: Resource materialities". Anthropological Quarterly 87 (1): 5-30. http://www.jstor.org/stable/43652719

Rose, Deborah Bird. 2017. "Shimmer: when all you love is being trashed". En Arts of living on a damaged planet, editado por Anna Tsing, Heather Swanson, Elaine Gan y Nils Bubandt, G51-63. Minneapolis: University of Minnesota Press.

Sapir, Edward. 1944. “Grading, a study in semantics”. Philosophy of Science 11 (2): 93-116. https://www.jstor.org/stable/184355

Schieffelin, Bambi, Kathryn Woollard y Paul Kroskrity, eds. (1998) 2012. Ideologías lingüisticas. Práctica y teoría. Madrid: Catarata.

Shever, Elana. 2020. "Becoming stone: on the coming-into-being of fossils in the American West”. Anthropological Quarterly 93 (3): 461-495. https://doi.org/10.1353/anq.2020.0055

Silverstein, Michael. 2004. “'Cultural' concepts and the language-culture Nexus1”. Current Anthropology 45 (5): 621-652. https://doi.org/10.1086/423971

Silverstein, Michael y Greg Urban, eds. 1996. Natural histories of discourse. Chicago: University of Chicago Press.

Simondon, Gilbert. 2009. La individuación. A la luz de las nociones de forma y de información. Buenos Aires: Cactus.

Stewart, Kathleen. 2007. Ordinary affects. Durham: Duke University Press.

Suárez Guava, Luis Alberto. 2013. "Guacas. Teorías del mundo en los Andes colombianos”. Mора Mора 1 (22): 10-49. https://revistas.udenar.edu.co/index.php/rmopa/article/ view/2975

Taussig, Michael. 2008. “Redeeming indigo”. Theory, Culture \& Society 25 (3): 1-15. https://doi. org/10.1177/0263276408090655

Tsing, Anna. 2013. "Sorting out commodities: how capitalist value is made through gifts". HAU: Journal of Ethnographic Theory 3 (1): 21-43. https://doi.org/10.14318/hau3.1.003

-. 2015. The mushroom at the end of the world: on the possibility of life in capitalist ruins. Princeton: Princeton University Press.

Tsing, Anna y Paulla Ebron. 2015. "Writing and rhythm: call and response with Anna Tsing and Paulla Ebron”. Journal of the Royal Anthropological Institute 21 (3): 683-687. https://doi. org/10.1111/1467-9655.12256

Tsing, Anna, Heather Swanson, Elaine Gan y Nils Bubandt, eds. 2017. Arts of living on a damaged planet. Minneapolis: University of Minnesota Press. 
Uribe, María Victoria. 1992. Limpiar la tierra. Guerra y poder entre esmeralderos. Bogotá: Cinep.

Valero, Helí. 2008. "El río Minero: matagente, ladrón y fantasmal”. Maguaré 22: 205-222. https://revistas.unal.edu.co/index.php/maguare/article/view/15291

Vasco, Luis Guillermo. 2002. Entre selva y páramo: viviendo y pensando la lucha india. Bogotá: Instituto Colombiano de Antropología e Historia.

Walsh, Andrew. 2004. "In the wake of things: speculating in and about sapphires in Northern Madagascar”. American Anthropologist 106 (2): 225-237. https://doi.org/10.1525/ aa.2004.106.2.225

Young, Diana. 2018. “Color”. The International Encyclopedia of Anthropology, 1-6. https://doi. org/10.1002/9781118924396.wbiea2210 